\title{
Universal dephasing in a chiral 1D interacting fermion system
}

\author{
Clemens Neuenhahn and Florian Marquardt \\ Department of Physics, Arnold Sommerfeld Center for Theoretical Physics, and Center for NanoScience, \\ Ludwig Maximilians Universität München, Theresienstr. 3\%, 80333 Munich, Germany
}

\begin{abstract}
We consider dephasing by interactions in a one-dimensional chiral fermion system (e.g. a Quantum Hall edge state). For finite-range interactions, we calculate the spatial decay of the Green's function at fixed energy, which sets the contrast in a Mach-Zehnder interferometer. Using a physically transparent semiclassical ansatz, we find a power-law decay of the coherence at high energies and zero temperature $(T=0)$, with a universal asymptotic exponent of 1 , independent of the interaction strength. We obtain the dephasing rate at $T>0$ and the fluctuation spectrum acting on an electron.
\end{abstract}

Studying the loss of quantum coherence is important both for fundamental reasons (quantum-classical transition, measurement process, equilibration) and with regard to possible applications of quantum mechanics (interferometry, quantum information processing).

Dephasing of electrons in Luttinger liquids is interesting as an example of a non-perturbative, strongly correlated model case [1, 2, 3, 4, 5, 6]. In contrast, the situation for (spinless) chiral interacting fermion systems, such as edge states in the integer quantum Hall effect (QHE), seems to be clear. Within the standard ansatz of pointlike interactions, an interacting chiral model is only a Fermi gas with a renormalized velocity. Recently though it was realized that such models may present interesting physics if finite-range interactions are considered [7] (cf. also [8]). This research is motivated by recent studies of dephasing in QHE Mach-Zehnder interferometers, both by intrinsic interactions 7, 9, 10, 11, 12, 13, 14] and external baths 11, 15, 16, 17, 18, 19]. Remarkable experiments [19, 20, 21, 22, 23] have revealed novel effects at high bias voltages, which is the regime we are going to study.

At low energies and temperatures, chiral interacting fermions form a Fermi liquid and are fully coherent at $T=0$ and $\epsilon=\epsilon_{F}$. It was found that the features at intermediate energies depend on the details of the interaction potential [7, 8, 24]. However, here we study the coherence of interacting chiral fermions at high energies (higher than the cutoff for the interaction potential). Our central result is that (at $T=0$ ) there is a universal power-law decay of coherence with propagation distance, where the exponent is independent of interaction strength. This is in contrast to physical expectation, where decoherence should grow with increasing coupling. We identify the reason behind this as a subtle cancellation between increasing interaction strength and decreasing density fluctuations in the sea of other electrons. We will derive this first within a semiclassical ansatz that is later shown to be exact at high energies, comparing it to bosonization. We will discuss deviations from the leading behaviour and the situation at $T>0$. The result is particularly remarkable since usually universal behaviour is confined to the low-energy regime.
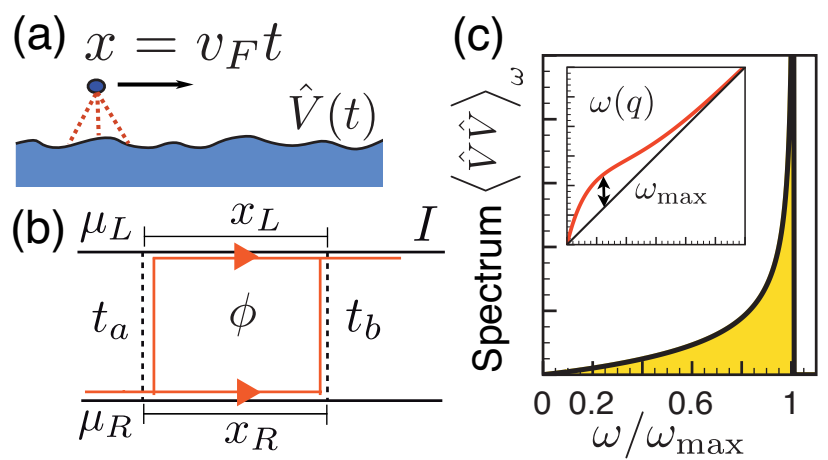

Figure 1: (a) A single electron propagating at high energies feels a fluctuating potential $\hat{V}(t)$, as it interacts with the sea of other electrons. (b) Scheme of the Mach-Zehnder interferometer setup. (c) The fluctuation spectrum (at $T=0$ and $\alpha>0)$. Inset: plasmonic dispersion relation.

The model. - We consider fermions in one dimension, propagating chirally at speed $v_{F}$ and interacting via a potential $U\left(x-x^{\prime}\right)$ :

$$
\begin{aligned}
\hat{H}= & \sum_{k} v_{F} k \hat{c}_{k}^{\dagger} \hat{c}_{k}+ \\
& \frac{1}{2} \int d x d x^{\prime} \hat{\psi}^{\dagger}(x) \hat{\psi}^{\dagger}\left(x^{\prime}\right) U\left(x-x^{\prime}\right) \hat{\psi}\left(x^{\prime}\right) \hat{\psi}(x),(1)
\end{aligned}
$$

where $\hat{\psi}(x)=L^{-1 / 2} \sum_{k} \hat{c}_{k} e^{i k x}$ are the fermion operators, the normalization volume $L$ tends to infinity in the end, $k \in 2 \pi L^{-1} \mathbb{Z}$, and $k \geq k_{c}$, with a cutoff $k_{c}$ that drops out of the results. We have set $\hbar=1$. After bosonization, the Hamiltonian is diagonal:

$$
\hat{H}=\sum_{q>0} \omega(q) \hat{b}_{q}^{\dagger} \hat{b}_{q}+\mu \hat{N}
$$

The bosonic operators $\hat{b}_{q}$ of Eq. (2) describe the density fluctuations $\hat{\rho}(x) \equiv \hat{\psi}^{\dagger}(x) \hat{\psi}(x)-\bar{\rho}$, where $\bar{\rho}$ is the mean density:

$$
\hat{\rho}(x)=\sum_{q>0} \sqrt{\frac{q}{2 \pi L}}\left(\hat{b}_{q} e^{i q x}+\text { h.c. }\right) .
$$


The plasmonic dispersion relation depends on the interaction potential's Fourier transform, $U_{q}=$ $\int d x e^{-i q x} U(x)$ :

$$
\omega(q)=v_{F} q\left[1+\frac{U_{q}}{2 \pi v_{F}}\right] .
$$

Here $v_{F}$ is the velocity at $q \rightarrow \infty$ and we define $\tilde{v} \equiv$ $v_{F}+U_{q \rightarrow 0} /(2 \pi)=v_{F}(1+\alpha)$. The dimensionless interaction strength is $\alpha \equiv U_{0} /\left(2 \pi v_{F}\right)$. $U_{q}$ is assumed to decay beyond some scale $q_{c}$.

Interferometry. - To probe the electrons' coherence, we imagine an electronic Mach-Zehnder interferometer [Fig.11(b)] [9, 20], i.e. two chiral wires connected by small tunnel couplings $t_{a}$ and $t_{b}$ at two "beam splitters" (quantum point contacts). This permits to express the current to leading order in the tunnel coupling [7, 12], via the single-particle Green's functions (GF) in the wires. This is possible under the assumption that there are no interactions between the wires (and therefore no vertex corrections in the result), which is reasonable due to their spatial separation. The quantity of interest is the visibility $\mathcal{V}$, i.e. the contrast of the current interference pattern that is displayed when changing the magnetic flux $\phi$. We define $\mathcal{V} \equiv\left(I_{\max }-I_{\min }\right) /\left(I_{\max }+I_{\min }\right)$, where $I_{\max }=\max _{\phi} I(\phi)$. In contrast to [7], we write $\mathcal{V}$ in terms of the GF in energy-space: $G^{>}(x, \epsilon)$ is the Fourier transform of $G^{>}(x, t)=-i\left\langle\hat{\psi}(x, t) \hat{\psi}^{\dagger}(0,0)\right\rangle$. It gives the amplitude for an electron injected at energy $\epsilon$ to propagate coherently a distance $x$. This yields (at $T=0)$ :

$$
\mathcal{V}=\frac{2\left|t_{a} t_{b}^{*}\right|}{\left|t_{a}\right|^{2}+\left|t_{b}\right|^{2}} \cdot \frac{\left|\int_{0}^{\delta \mu} d \epsilon G_{L}^{>}\left(x_{L}, \epsilon\right) G_{R}^{<}\left(-x_{R}, \epsilon-\delta \mu\right)\right|}{(2 \pi)^{2} \int_{0}^{\delta \mu} d \epsilon \nu_{L}(\epsilon) \nu_{R}(\epsilon-\delta \mu)}
$$

There are contributions from all electrons inside the voltage interval, $\epsilon=0 \ldots \delta \mu$, where $\delta \mu=\mu_{L}-\mu_{R}=q_{e} V>0$ is the bias between the left $(\mathrm{L})$ and the right $(\mathrm{R})$ interferometer arm. $G_{L, R}$ are the bulk GF's [at $\mu=0$ ] for particles $(>)$ and holes $(<)$, where $G^{<}(x, \epsilon)=G^{>*}(x,-\epsilon)$. At $T=0$ one obtains the tunneling density of states from $2 \pi \nu(\epsilon)=\left|G^{>}(x=0, \epsilon)\right|+\left|G^{<}(0, \epsilon)\right|$. For $x_{L}=x_{R}=x$, the decay of visibility is thus determined by the GF decay to be discussed in the following.

Decoherence of a high-energy electron. - We employ a physically intuitive semiclassical ansatz for the GF's, that becomes exact in the limit of high energies, as we will confirm later by comparing it to bosonization. Electrons at high energies $\epsilon \gg v_{F} q_{c}$ propagate at the speed $v_{F}$. Scattering by a few multiples of $q_{c}$ will not bring them near the Fermi energy, so Pauli blocking is unimportant. The visibility at high bias voltage is dominated by these electrons. The sea of other electrons produces a fluctuating potential $\hat{V}(t)$ acting on such a high-energy electron at its classical position $x=v_{F} t$. It is obtained by convoluting the density with the interaction potential [Fig. 1(a)]:

$$
\hat{V}(t)=\int d x^{\prime} U\left(x^{\prime}-v_{F} t\right) \hat{\rho}\left(x^{\prime}, t\right) .
$$

As known from bosonization, the fluctuations of $\hat{\rho}$ are purely Gaussian. The ansatz assumes the electron to pick up a random phase from the potential fluctuations $\hat{V}(t)$. As a result, its non-interacting GF $G_{0}^{>}$is multiplied by the average of the corresponding phase factor: $G^{>}(x, \epsilon)=G_{0}^{>}(x, \epsilon) \cdot \exp (-F(x))$, where

$$
\begin{aligned}
e^{-F(x)} & \equiv\left\langle\hat{T} \exp \left[-i \int_{0}^{x / v_{F}} d t^{\prime} \hat{V}\left(t^{\prime}\right)\right]\right\rangle \\
& =\exp \left[-\frac{1}{2} \int_{0}^{x / v_{F}} d t_{1} d t_{2}\left\langle\hat{T} \hat{V}\left(t_{1}\right) \hat{V}\left(t_{2}\right)\right\rangle\right]
\end{aligned}
$$

depends on the propagation distance $x$, but turns out to be energy-independent in the high-energy limit discussed here. A related approach was introduced both for electron dephasing in 1D ballistic wires by an external quantum environment [16, 18], and for describing two coupled (non-chiral) Luttinger liquids [3] or 1D systems with a nonlinear dispersion relation [25, 26]. The form of $e^{-F(x)}$ is exactly the same as that for pure dephasing of a qubit by quantum noise [11, 16, 18]. The decay is determined by the fluctuation spectrum in the electron's frame of reference, $\langle\hat{V} \hat{V}\rangle_{\omega}=\int d t e^{i \omega t}\langle\hat{V}(t) \hat{V}(0)\rangle$. The magnitude of the GF (i.e. the electron's coherence) turns out to decay as

$\frac{\left|G^{>}(x, \epsilon)\right|}{\left|G_{0}^{>}(x, \epsilon)\right|}=\exp \left[-\int_{-\infty}^{+\infty} \frac{d \omega}{2 \pi} \frac{\sin ^{2}\left(\omega x / 2 v_{F}\right)}{\omega^{2}}\langle\{\hat{V}, \hat{V}\}\rangle_{\omega}\right]$,

where $\langle\{\hat{V}, \hat{V}\}\rangle_{\omega}=\langle\hat{V} \hat{V}\rangle_{\omega}+\langle\hat{V} \hat{V}\rangle_{-\omega}$ denotes the symmetrized spectrum and $\left|G_{0}^{>}(x, \epsilon)\right|$ is constant in the highenergy regime. From Eq. (6), we obtain for the potential spectrum

$$
\langle\hat{V} \hat{V}\rangle_{\omega}=\int \frac{d q}{2 \pi}\left|U_{q}\right|^{2}\langle\hat{\rho} \hat{\rho}\rangle_{q, \omega+v_{F} q},
$$

which derives from the Galileo-transformed spectrum of the density fluctuations. We first focus on $T=0$, where $\langle\hat{\rho} \hat{\rho}\rangle_{q, \omega}=\theta(q) q \delta(\omega-\omega(q))$. The spectrum has two distinct features (cf. Fig. 1(c)).

At high frequencies, we obtain a singularity $\langle\{\hat{V}, \hat{V}\}\rangle_{\omega}^{T=0} \propto 1 / \sqrt{\omega_{\max }-|\omega|}$ at the cutoff frequency $\omega_{\max }=\max \left(\omega(q)-v_{F} q\right)$, which is the maximum frequency in the Galileo-transformed plasmon dispersion relation. This singularity arises since $\omega(q) \approx \omega\left(q^{*}\right)+$ $\omega^{\prime \prime}\left(q^{*}\right) \cdot\left(q-q^{*}\right)^{2} / 2$ in the vicinity of $q^{*}$, where $\omega\left(q^{*}\right)=$ $v_{F} q^{*}+\omega_{\max }$.

At low frequencies $\omega \ll v_{F} q_{c}$, the spectrum increases linearly in $\omega$, corresponding to "Ohmic" noise, which is 
ubiquitous in many contexts [27]. Here, it derives from the interaction with $1 \mathrm{D}$ sound waves (plasmons). For potentials that are smooth in real space (i.e. all the moments of $\left|U_{q}\right|$ are finite), the leading low- $\omega$ behaviour is determined by small $q$ in Eq. (9). The result is :

$$
\langle\{\hat{V}, \hat{V}\}\rangle_{\omega}^{T=0}=\frac{U_{q \rightarrow 0}^{2}}{\left(\tilde{v}-v_{F}\right)^{2}} \frac{|\omega|}{2 \pi}=2 \pi|\omega| .
$$

The prefactor of the spectrum turns out to be independent of the coupling strength $\alpha$. This is in contrast to non-chiral Luttinger liquids, where an Ohmic spectrum has been found with an interaction-dependent prefactor [3]. An increase in interaction strength is canceled by stiffening the density fluctuations, i.e. shifting them to higher frequencies in the co-moving frame, and thereby decreasing their magnitude. This translates into a universal power-law decay for the GF at large $x$ :

$$
\left|G^{>}(x, \epsilon)\right| \propto \frac{1}{x^{1}} .
$$

More precisely, we claim that asymptotically the exponent becomes 1: $\lim _{x \rightarrow \infty}-\ln \left|G^{>}(x, \epsilon)\right| / \ln x=1$. While here the cancellation of $\alpha$ is unexpected, a similar effect is known for Nyquist noise, where the electron charge cancels at low $\omega$ due to screening. Note the contrast to dephasing by an external bath, where the decay gets weaker for lower coupling, and also to the coupling-dependent exponents in a Luttinger liquid. This central result is illustrated in Fig. 2, based on Eq. (8). The power-law decay reflects the Anderson orthogonality catastrophe, where the many-body state of the 'other' electrons evolves depending on the path of the given electron. The oscillations are due to the cutoff in $\langle\hat{V} \hat{V}\rangle_{\omega}$. Its amplitude depends on $\alpha$ (see below), but it vanishes for large $x$. These oscillations can be understood as 'coherence revivals', where the entanglement with the environment is partly undone at certain times, in the manner of 'quantum eraser' experiments.

In order to understand how the non-interacting limit is recovered ( $\alpha=0$, where $\left|G^{>}(x, \epsilon)\right|$ is constant as a function of $x$ ), we have to discuss its range of validity. As the linear slope in the spectrum applies only at $|\omega| \ll \omega_{\max }$, we must require $\omega_{\max } x / v_{F} \gg 1$. Since $\omega_{\max }$ vanishes with $\alpha$, the limiting regime is reached at ever larger values of $x$ for $\alpha \rightarrow 0$.

We now discuss the deviations from the leading low$\omega$ behaviour in $\langle\{\hat{V}, \hat{V}\}\rangle_{\omega}$. These are due to the contributions from large $q$ in (9). E.g. a potential $U_{q}=U_{0} e^{-\left(|q| / q_{c}\right)^{s}}$ yields a sub-leading contribution $\langle\{\hat{V}, \hat{V}\}\rangle_{\omega}^{(s u b)}=2 \pi|\omega| /\left(s \ln \left(|\alpha| v_{F} q_{c} /|\omega|\right)\right)$. This turns into a term $s^{-1} \ln \left(\ln \left(|\alpha| q_{c} x\right)\right)$ in $F(x)$, yielding

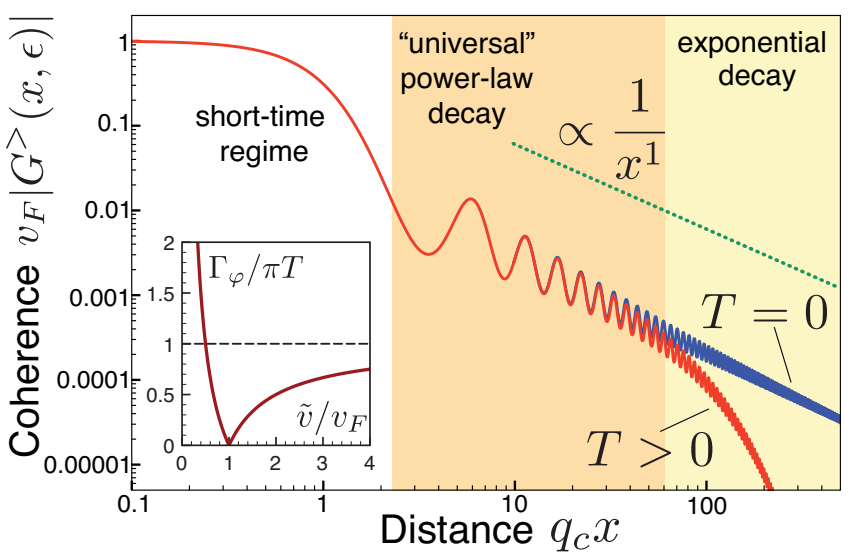

Figure 2: The coherence of an electron propagating at high energies in an interacting chiral system, as a function of propagation distance. The non-interacting case would be $v_{F}\left|G^{>}(x, \epsilon)\right| \equiv 1$. The asymptotic exponent for the powerlaw decay is universally given by 1 (see dashed line). At $T>0$, one obtains an exponential decay for large $x$, with a decay rate $\Gamma_{\varphi}$ (inset). The potential was $U_{q}=U_{0} e^{-\left|q / q_{c}\right|}$ with $U_{0} / v_{F}=2 \pi \alpha=20$, and $T / q_{c} v_{F}=0.01$.

a slow logarithmic decay of the prefactor in Eq. (11) that can be understood as an asymptotically vanishing correction $s^{-1} \ln \left(\ln \left(|\alpha| q_{c} x\right)\right) / \ln (x) \rightarrow 0$ to the exponent 1 . The subleading oscillatory contribution to $F$ is $-C_{s} \sin \left(\omega_{\max } x / v_{F}+\pi / 4\right) / \sqrt{2 \pi|\alpha| q_{c} x}$, with a numerical prefactor $C_{s}$ [e.g. $C_{1}=2 \pi \sqrt{e}$. In contrast, consider a potential that is non-smooth (i.e. $\int d q\left|U_{q}\right| q^{n}$ does not converge for some $n$ ). If $U_{q}=u q^{-n}$ for large $q$, then we find an additional contribution $2 \pi|\omega|(n-1)^{-1}[n>1]$. It modifies the leading behaviour of $\langle\{\hat{V}, \hat{V}\}\rangle_{\omega}$ and changes the decay into $\left|G^{>}(x, \epsilon)\right| \propto 1 / x^{1+1 /(n-1)}$. The universal exponent is recovered as $n \rightarrow \infty$.

For $T>0$, the large- $x$ limit yields an exponential decay $\left|G^{>}(x, \epsilon)\right| \propto \exp \left[-\Gamma_{\varphi} x / v_{F}\right]$, with

$$
\Gamma_{\varphi}=\pi T\left|1-\frac{v_{F}}{\tilde{v}}\right|=\pi T\left|1+\alpha^{-1}\right|^{-1}
$$

For $\alpha \rightarrow 0$, this rate vanishes as $\Gamma_{\varphi}=\pi T|\alpha|$, i.e. it is non-analytic in $U_{0} \propto \alpha$. Dephasing rates linear in $T$ have also been found in non-chiral Luttinger liquids [3, 4, 5]. At large repulsion, $U_{0} \rightarrow+\infty$, we have the universal result $\Gamma_{\varphi} \rightarrow \pi T$. For attractive interaction, $\Gamma_{\varphi}$ diverges at the instability for $\alpha \rightarrow-1$, where $\tilde{v} \rightarrow 0$ gives rise to thermally excited low-frequency modes.

Contrast this behaviour against pure dephasing of a qubit by Nyquist noise. There, a power-law decay $t^{-\gamma}$ at $T=0$ implies a decay rate $\Gamma_{\varphi}=\pi \gamma T$ for $T>0$. In the present case, the Galileo transformation turns the lab-frame temperature $T$ into $T_{\text {eff }}$ in the co-moving frame. We find $T_{\text {eff }}=T\left|1-v_{F} / \tilde{v}\right|$ enters in the fluctuation-dissipation theorem relation $\langle\{\hat{V}, \hat{V}\}\rangle_{\omega}^{T}=$ $\left(2 T_{\text {eff }} /|\omega|\right)\langle\{\hat{V}, \hat{V}\}\rangle_{\omega}^{T=0}$. Only for large repulsion, we get 


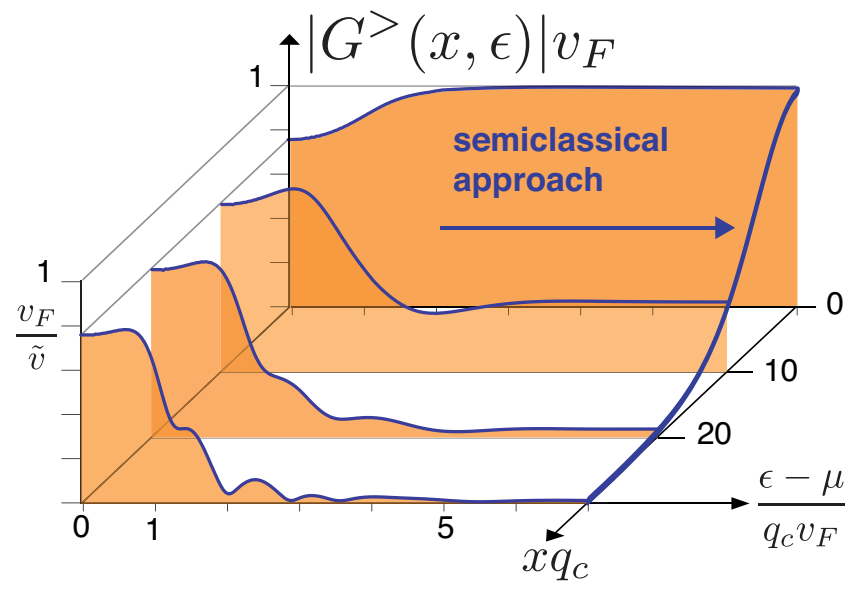

Figure 3: The evolution of the Green's function with energy $\epsilon$ of the injected electron at $T=0$, for various propagation distances, according to bosonization [Eq. (13)]. The curve at the right corresponds to the semiclassical ansatz [Eq. (8)], which is exact for high energies, as is evident in the figure. The potential was $U_{q}=U_{0} e^{-\left(q / q_{c}\right)^{2}}$ with $U_{0} / v_{F}=2 \pi \alpha=2$.

$T_{\text {eff }} \rightarrow T$, and the universal power-law for $T=0$ turns into a universal decay rate for $T>0$.

Green's function from bosonization. - We employ the standard connection [28, 29] between the bosonic phase field $\hat{\Phi}(x)=i \sum_{q>0} \sqrt{\frac{2 \pi}{L q}} e^{-a q}\left[\hat{b}_{q}(t) e^{i q x}-h . c.\right]$ and the fermion operators $\hat{\psi}(x)=\frac{\hat{F}}{\sqrt{2 \pi a}} e^{i k_{F} x} e^{-i \hat{\Phi}(x)}$ (where $\hat{b}_{q}(t)=\hat{b}_{q}(0) e^{-i \omega_{q} t}, \hat{F}$ is the Klein factor, and $a \rightarrow 0$ provides the regularization at short distances). This yields the GF

$$
\begin{aligned}
G^{>}(x, t)= & \frac{-i}{2 \pi a} e^{-i \mu\left(t-x / v_{F}\right)} . \\
& \exp \left[\langle\hat{\Phi}(x, t) \hat{\Phi}(0,0)\rangle-\left\langle\hat{\Phi}(0,0)^{2}\right\rangle\right]
\end{aligned}
$$

A numerical Fourier transform produces $G^{>}(x, \epsilon)$ (see Fig.(3). The dip near $\epsilon=0$ in the tunneling density $\propto\left|G^{>}(x=0, \epsilon)\right|$ is due to the renormalization of the velocity. The decay of the GF with increasing $x$ is due to interaction-induced decoherence. Most importantly, the decay at high energies (i.e. $\epsilon-\mu \gg v_{F} q_{c}, \omega_{\max }$ ) is reproduced exactly by the semiclassical approach (see Fig. 33). This may be understood as follows: Evaluation of (13) produces a broad, dispersing peak [7] moving with the renormalized velocity $\tilde{v}$. There is another, sharp peak at $x=v_{F} t$. This is due to contributions from high frequencies in the plasmon dispersion, and the evolution of its weight determines the decay of $G^{>}(x, \epsilon)$ at high energies. That weight can be obtained from bosonization(13), evaluated at $x=v_{F} t$, which turns out to be identical to the semiclassical ansatz in Eq. (8).

In interferometry, these universal results determine the visibility $\mathcal{V}$ for high bias voltage. At $T=0$ we obtain a decay $\mathcal{V} \propto 1 / x^{2}$ independent of $\delta \mu$ at high bias (note that $\mathcal{V} \rightarrow 1$ for $\delta \mu \rightarrow 0$, as expected [7]), and the exponential decay for $T>0$ is transferred to $\mathcal{V}$ as well.

Conclusions. - The coherence of an electron moving in a chiral system obeys a universal asymptotic powerlaw decay at $T=0$, with an exponent 1 independent of interaction strength, for energies above the scale set by the interaction range. For $T>0$, the decay rate becomes coupling-dependent except in the limit of high couplings, where it reduces to a universal decay rate $\Gamma_{\varphi}=\pi T$. These results were derived by a physically transparent semiclassical approach that is exact in the high-energy limit.

Acknowledgements. - We thank J. Chalker, Y. Gefen, and O. Yevtushenko for fruitful discussions. Financial support by DIP, NIM, the Emmy-Noether program and the SFB/TR 12 is gratefully acknowledged.

[1] W. Apel and T. M. Rice, Journal of Physics C: Solid State Physics 16, L271 (1983).

[2] K. Le Hur, Phys. Rev. B 65, 233314 (2002).

[3] K. L. Hur, Physical Review Letters 95, 076801 (2005).

[4] I. V. Gornyi, A. D. Mirlin, and D. G. Polyakov, Physical Review Letters 95, 046404 (2005).

[5] K. L. Hur, Physical Review B 74, 165104 (2006).

[6] D. Gutman, Y. Gefen, and A. Mirlin, arXiv:0804.4294v1 (2008).

[7] J. T. Chalker, Y. Gefen, and M. Y. Veillette, Physical Review B 76, 085320 (pages 11) (2007).

[8] V. Meden and K.Schönhammer, Phys. Rev. B 47, 16205 (1993).

[9] G. Seelig and M. Büttiker, Phys. Rev. B 64, 245313 (2001).

[10] K. T. Law, D. E. Feldman, and Y. Gefen, Phys. Rev. B 74, 045319 (2006).

[11] I. Neder and F. Marquardt, New Journal of Physics 9, 112 (2007).

[12] E. V. Sukhorukov and V. V. Cheianov, Phys. Rev. Lett. 99, 156801 (2007).

[13] I. Neder and E. Ginossar, Physical Review Letters 100, 196806 (2008).

[14] I. P. Levkivskyi and E. V. Sukhorukov, Physical Review B 78, 045322 (2008).

[15] F. Marquardt and C. Bruder, Phys. Rev. Lett. 92, 056805 (2004).

[16] F. Marquardt, Europhysics Letters 72, 788 (2005).

[17] H. Förster, S. Pilgram, and M. Büttiker, Phys. Rev. B 72, 075301 (2005).

[18] F. Marquardt, Phys. Rev. B 74, 125319 (2006).

[19] I. Neder, F. Marquardt, M. Heiblum, D. Mahalu, and V. Umansky, Nature Physics 3, 534 (2007).

[20] Y. Ji, Y. Chung, D. Sprinzak, M. Heiblum, D. Mahalu, and H. Shtrikman, Nature 422, 415 (2003).

[21] I. Neder, M. Heiblum, Y. Levinson, D. Mahalu, and V. Umansky, Phys. Rev. Lett. 96, 016804 (2006).

[22] L. Litvin, A. Helzel, H. Tranitz, W. Wegscheider, and C. Strunk, Phys. Rev. B 78, 075303 (2008).

[23] P. Roulleau, F. Portier, P. Roche, A. Cavanna, G. Faini, 
U. Gennser, and D. Mailly, Physical Review Letters 100, 126802 (2008).

[24] V. Meden, Phys. Rev. B 60, 4571 (1999).

[25] M. Pustilnik, M. Khodas, A. Kamenev, and L. I. Glazman, Physical Review Letters 96, 196405 (2006).

[26] A. Imambekov and L. I. Glazman, Physical Review Letters 100, 206805 (2008).
[27] U. Weiss, Quantum Dissipative Systems (World Scientific, Singapore, 2000).

[28] J. v. Delft and H. Schoeller, Annalen der Physik 4, 225 (1998).

[29] T. Giamarchi, Quantum physics in one dimension (Oxford University Press, 2006). 